\title{
Éditorial
}

\section{Réduire la mortalité prématurée chez les jeunes adultes et les adultes d'âge moyen}

\author{
Joel G. Ray, M.D., FRCPC $(1,2,3)$
}

Diffuser cet article sur Twitter

Le décès d'un individu au début ou au milieu de sa vie adulte est un événement inattendu dont les conséquences tragiques touchent à la fois les parents de la personne décédée, les membres de sa fratrie, son conjoint ou sa conjointe, ses enfants ${ }^{1-3}$ et ses amis. La prévention des décès prématurés est un objectif central des programmes de soins de santé et de santé publique, et plus largement de la société.

La mortalité prématurée correspond à l'espérance de vie non atteinte. Les définitions conventionnelles de la mortalité prématurée et des années potentielles de vie perdues $^{4}$, qui s'appliquent à l'ensemble de la population de la naissance jusqu'à l'âge de $65^{5}$ ou de $75^{6}$ ans, nuisent à notre compréhension des facteurs associés aux décès évitables à l'âge adulte. Par exemple, les décès qui surviennent durant l'enfance touchent surtout les nourrissons - du fait des malformations congénitales et des naissances prématurées. À l'autre extrémité du cycle de vie, chez les aînés de 65 à 75 ans (chez qui l'on compte la plus grande proportion des décès), ce sont les décès par cancer et par maladies cardiovasculaires qui dominent. Pour l'ensemble des Canadiens, les cinq principales causes de décès sont le cancer (30 \%), les maladies cardiaques (21\%), les accidents vasculaires cérébraux (6\%), les maladies du poumon (5\%) et les blessures non intentionnelles $(4 \%)^{7}$. Chez les Canadiens de 25 à 34 ans, ce sont les blessures non intentionnelles (29\%), le suicide (20\%), le cancer (12\%), les maladies cardiaques (5\%) et les homicides $(5 \%)^{7}$. Chez les Canadiens de 35 à 44 ans, parmi les cinq principales causes de décès, on compte les blessures non intentionnelles, le suicide et les maladies du foie, ces dernières découlant souvent de la consommation excessive d'alcool et de l'usage de drogues injectables. À Toronto, les causes de décès prématuré suivent la même tendance ${ }^{8}$.

De l'ensemble des décès survenant chez les Canadiens de 20 à 64 ans, $20 \%$ touchent ceux de 20 à 44 ans $^{9}$. De plus, la plupart des décès prématurés chez les jeunes adultes et les adultes d'âge moyen sont vraiment évitables. Au Canada, environ $6 \%$ de ces décès sont liés à l'alcool deux fois plus fréquemment chez les hommes $(7,6 \%)$ que chez les femmes $(3,5 \%)^{10}$. En Russie, où la consommation d'alcool constitue un problème de santé publique majeur, jusqu'à $43 \%$ des décès signalés chez les hommes de 25 à 54 ans sont attribuables à une consommation d'alcool dangereuse ${ }^{11}$. En Ontario, en 2010, un décès sur huit chez les adultes de 25 à 34 ans était lié à la consommation d'opioïdes $^{12}$ et, à l'échelle du pays, nous constatons l'émergence d'une épidémie liée à l'usage d'opioïdes qui a ravagé et emporté la vie de nombre de Canadiens.

La maladie mentale et les comportements criminels sont également interconnectés dans leurs effets sur la mortalité prématurée. Selon deux vastes études suédoises menées auprès de 15337 adultes atteints d'un trouble bipolaire, qui ont été appariés selon l'âge et le sexe à 20 adultes choisis aléatoirement au sein de la population générale, $22 \%$ des sujets commettaient des actes suicidaires ou criminels après leur diagnostic de trouble bipolaire, contre $4,6 \%$ des membres de la population générale (risque relatif $[R R]$ corrigé $=3,0$; intervalle de confiance [IC] à $95 \%: 2,9$ à $3,2)^{13}$. Les personnes atteintes d'un trouble bipolaire présentaient un risque de suicide 14,6 fois plus élevé (IC à $95 \%$ : 12,1 à $17,6)$, en particulier celles qui présentaient des antécédents de tentative de suicide ou un trouble lié à la consommation d'alcool ou de drogues ${ }^{13}$. Dans une étude menée auprès de 475 garçons délinquants et de 456 garçons non délinquants suivis de leurs 14 ans à leurs 65 ans, respectivement $6,1 \%$ et $2,4 \%$ sont décédés de causes non naturelles avant l'âge de 40 ans. Les comportements antisociaux chez les jeunes et l'abus d'alcool se sont révélés des prédicteurs de ce résultat, et les décès étaient davantage susceptibles de résulter d'un homicide et d'une piètre autogestion de la santé $^{14}$. Dans une étude menée auprès de récidivistes en Finlande, le risque de décès avant l'âge de 30 ans était 29 fois plus élevé que chez les personnes n'ayant jamais commis d'acte criminel ${ }^{15}$. Sur l'ensemble des individus emprisonnés dans un établissement correctionnel provincial canadien, le ratio normalisé de mortalité s'établit à 4,0 (IC à $95 \%: 3,9$ à 4,1 ), les blessures et l'empoisonnement représentant $38 \%$ de tous les décès ${ }^{16}$, et les RR de décès les plus importants sont observés chez les jeunes délinquants, en particulier chez les femmes ${ }^{16}$. Des statistiques similaires ont été relevées chez les personnes emprisonnées dans un établissement correctionnel fédéral ${ }^{17}$. Il appert donc que certains adultes susceptibles de mourir prématurément sont aux prises avec des problèmes de santé mentale, de toxicomanie et de criminalité, dont l'origine remonte souvent à leur jeunesse.

Rattachement de l'auteur :

1. Department of Medicine, St. Michael's Hospital, University of Toronto, Toronto (Ontario), Canada

2. Department of Obstetrics and Gynecology, St. Michael's Hospital, University of Toronto, Toronto (Ontario), Canada

3. Institute of Health Policy, Management and Evaluation, St. Michael's Hospital, University of Toronto, Toronto (Ontario), Canada

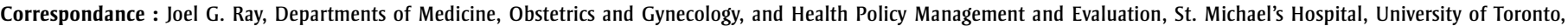
30, rue Bond, Toronto (Ontario) M5B 1W8; tél. : 416-864-6060, poste 77442; téléc. : 416-864-5485; courriel : rayj@smh.ca 
Il existe des " facteurs de risque génériques » de mortalité prématurée de nature intentionnelle et non intentionnelle, en particulier des facteurs de risques concentrés autour de la maladie mentale. Neeleman a procédé à un examen systématique de 163 cohortes et a constaté que plusieurs facteurs de risque de suicide connus - en particulier des antécédents d'automutilation, d'abus d'alcool et de drogues et d'une maladie mentale grave - ont été également associés à des décès non liés au suicide ${ }^{18}$. Lai et ses collaborateurs ont évalué 22 études épidémiologiques sur la prévalence de la maladie psychiatrique chez les personnes souffrant de toxicomanie ${ }^{19}$ : les personnes qui consommaient des drogues illicites étaient plus susceptibles de souffrir d'une dépression majeure (risque 3,8 fois plus élevé (IC à $95 \%: 3,0$ à 4,8]) et de présenter un trouble anxieux (risque 2,9 fois plus élevé [IC à $95 \%: 2,6$ à 3,3]) et les rapports de cotes chez les personnes ayant un trouble lié à la consommation d'alcool étaient respectivement de 2,4 (IC à $95 \%: 2,2$ à 2,6 ) et 2,1 (IC à $95 \%: 2,0$ à 2,2 ). Nous pouvons de ce fait utiliser ces facteurs de risque génériques, dont les antécédents d'automutilation, d'abus d'alcool et de drogues et d'une maladie mentale grave, pour déceler les adultes à risque de mourir prématurément, dont plusieurs pourraient faire l'objet d'une intervention, et ce, dès leur enfance ${ }^{18}$.

Ce n'est pas un hasard si l'augmentation de la prévalence de la dépendance à l'égard des aliments obésogènes hyper-savoureux ${ }^{20}$ et si l'émergence de l'obésité à l'échelle mondiale ${ }^{21}$ ont amené de nombreuses personnes à considérer l'obésité comme une maladie non transmissible, dont l'impact majeur sur la mortalité prématurée n’a pas encore été établi ${ }^{22}$. Les personnes prédisposées à la dépendance alimentaire ont également tendance à afficher des scores plus élevés aux questionnaires mesurant la dépression ${ }^{20}$ et sont également plus nombreuses à avoir été victimes de violence durant leur enfance ${ }^{23}$ et à avoir eu un accès plus limité à des installations facilitant l'activité physique, en particulier dans des régions où le statut socioéconomique est faible et au sein de certains groupes minoritaires $^{24}$, dont les enfants et les adolescents autochtones $^{25}$. Il est évident que la reconnaissance et la prise en compte adéquate de ces inégalités et de l'ensemble des autres inégalités peuvent aider les jeunes adultes à atteindre un indice de masse corporel plus sain, ce que Bhawra et ses collaborateurs $^{25}$, Frankish et ses collaborateurs ${ }^{26}$ et
Rao et ses collaborateurs ${ }^{27}$ soulignent dans ce numéro de Promotion de la santé et prévention des maladies chroniques au Canada.

Dans un autre article publié dans ce numéro, Steensma et ses collaborateurs présentent des données nationales sur l'espérance de vie ajustée en fonction de la santé (EVAS), une mesure composite non seulement de la quantité de vie, mais également de sa qualitée 28 . À l'échelle du Canada, on a montré qu'environ $45 \%$ de la variation de l'EVAS par région sanitaire s'expliquait par des différences liées au statut socioéconomique ${ }^{29}$, et Steensma et ses collaborateurs laissent entendre que la situation pourrait être pire à Terre-Neuveet-Labrador et à l'Île-du-Prince-Édouard, en particulier chez les hommes ${ }^{28}$. En réalité, il s'agirait d'une analyse conservatrice, compte tenu du fait que les données n'étaient disponibles que jusqu'en 2010 et que l'étude n'incluait pas les personnes vivant dans des réserves indiennes, dans certaines régions éloignées de l’Ontario et du Québec ainsi que dans les trois territoires canadiens - qui sont des zones où l'espérance de vie sans incapacité (une mesure semblable à l'EVAS) a tendance à être moins élevée ${ }^{29}$. Une modélisation de l'EVAS portant spécifiquement sur les personnes âgées de 20 à 45 ans peut certainement révéler dans quelle mesure certains facteurs qui ont un rôle sur les décès prématurés réduisent aussi parallèlement la qualité de vie au début et au milieu de la vie adulte.

Pour s'attaquer au problème de la mortalité prématurée chez les jeunes Canadiens et les Canadiens d'âge moyen, il faut d'abord définir clairement quelles personnes sont les plus à risque et quels sont les facteurs d'influence les plus probables, puis proposer des solutions adaptées, à la fois multidimensionnelles, réalistes et fondées sur des données probantes. Grâce aux études sur le traitement des maladies mentales et des dépendances, ainsi qu'à la prévention primaire et secondaire des blessures intentionnelles et non intentionnelles, nous devrions pouvoir non seulement réduire le nombre de décès prématurés au Canada, mais également améliorer le bien-être des personnes épargnées par cette triste destinée.

\section{Références}

1. Malkinson R, Bar-Tur L. Long term bereavement processes of older parents: the three phases of grief. Omega (Westport). 2004-2005;50(20):103-129.
2. Agerbo E. Midlife suicide risk, partner's psychiatric illness, spouse and child bereavement by suicide or other modes of death: a gender specific study. J Epidemiol Community Health. 2005; 59:407-412.

3. Guldin MB, Li J, Pedersen HS, Obel C, Agerbo E, Gissler M, Cnattingius S, Olsen J, Vestergaard M. Incidence of suicide among persons who had a parent who died during their childhood: a population-based cohort study. JAMA Psychiatry 2015;72(12):1227-1234.

4. Gardner JW, Sanborn JS: Years of potential life lost (YPLL) - what does it measure? Epidemiology. 1990;1: 322-329.

5. Eames M, Ben-Shlomo Y, Marmot MG. Social deprivation and premature mortality: regional comparison across England. BMJ. 1993;307(6912):10971102.

6. Wong MD, Shapiro MF, Boscardin WJ, Ettner SL. Contribution of major diseases to disparities in mortality. $\mathrm{N}$ Engl J Med. 2002;347(20):1585-1592.

7. Statistique Canada. Principales causes de décès au Canada - 2009. Ottawa (Ont.) : Statistique Canada; 2012. [ ${ }^{\circ}$ 84-215-X]

8. Matheson FI, Creatore MI, Gozdyra P, Park AL, Ray JG. A population-based study of premature mortality in relation to neighbourhood density of alcohol sales and cheque cashing outlets in Toronto, Canada. BMJ Open. 2014; 4(12):e006032.

9. Agence de la santé publique du Canada (ASPC). Rapport de l'administrateur en chef de la santé publique au Canada 2008. Ottawa (Ont) : ASPC; 2008. [n HP2-10/2008E]

10. Rehm J, Patra J, Popova S. Alcoholattributable mortality and potential years of life lost in Canada 2001: implications for prevention and policy. Addiction. 2006;101(3):373-384.

11. Leon DA, Shkolnikov VM, McKee M. Alcohol and Russian mortality: a continuing crisis. Addiction. 2009;104(10): 1630-1636. 
12. Gomes T, Mamdani MM, Dhalla IA, Cornish S, Paterson JM, Juurlink DN. The burden of premature opioid-related mortality. Addiction. 2014;109(9):14821488.

13. Webb RT, Lichtenstein P, Larsson H, Geddes JR, Fazel S. Suicide, hospitalpresenting suicide attempts, and criminality in bipolar disorder: examination of risk for multiple adverse outcomes. J Clin Psychiatry. 2014;75:e809-816.

14. Laub JH, Vaillant GE. Delinquency and mortality: a 50-year follow-up study of 1,000 delinquent and nondelinquent boys. Am J Psychiatry. 2000;157(1): 96-102.

15. Elonheimo H, Sillanmäki L, Sourander A. Crime and mortality in a population-based nationwide 1981 birth cohort: results from the FinnCrime study [Internet]. Crim Behav Ment Health. 2015 [consulté le 2 janvier 2017]. En ligne à : http://onlinelibrary .wiley.com/doi/10.1002/cbm.1973 /abstract

16. Kouyoumdjian FG, Kiefer L, Wobeser W, Gonzalez A, Hwang SW. Mortality over 12 years of follow-up in people admitted to provincial custody in Ontario: a retrospective cohort study. CMAJ Open 2016;4:E153-161.

17. Wobeser WL, Datema J, Bechard B, Ford P. Causes of death among people in custody in Ontario, 1990-1999. CMAJ. 2002;167(10):1109-1113.

18. Neeleman J. A continuum of premature death. Meta-analysis of competing mortality in the psychosocially vulnerable. Int J Epidemiol. 2001;30(1): 154-162.

19. Lai HM, Cleary M, Sitharthan T, Hunt GE. Prevalence of comorbid substance use, anxiety and mood disorders in epidemiological surveys, 1990-2014: A systematic review and meta-analysis. Drug Alcohol Depend. 2015;154:1-13.

20. Pursey KM, Stanwell P, Gearhardt AN, Collins CE, Burrows TL. The prevalence of food addiction as assessed by the Yale Food Addiction Scale: a systematic review. Nutrients. 2014;6(10): 4552-4590.
21. Lifshitz F, Lifshitz JZ. Globesity: the root causes of the obesity epidemic in the USA and now worldwide. Pediatr Endocrinol Rev. 2014;12(1):17-34.

22. Kontis V, Mathers CD, Bonita R, et al. Regional contributions of six preventable risk factors to achieving the $25 \times 25$ non-communicable disease mortality reduction target: a modelling study. Lancet Glob Health. 2015;3(12): e746-57.

23. Mason SM, Flint AJ, Roberts AL, Agnew-Blais J, Koenen KC, RichEdwards JW. Posttraumatic stress disorder symptoms and food addiction in women by timing and type of trauma exposure. JAMA Psychiatry. 2014;71(11): 1271-8.

24. Gordon-Larsen P, Nelson MC, Page P, Popkin BM. Inequality in the built environment underlies key health disparities in physical activity and obesity. Pediatrics. 2006;117(2):417-24.

25. Bhawra J, Cooke MJ, Guo Y, Wilk P. Relation entre l'obésité et la sécurité alimentaire des ménages, les caractéristiques des ménages et le milieu scolaire chez les enfants métis et des Premières Nations vivant hors réserve au Canada : résultats de l'Enquête auprès des peuples autochtones de 2012. Promotion de la santé et prévention des maladies chroniques au Canada. 2017;37(3):84-94.

26. Frankish CJ, Kwan B, Gray DE, Simpson A, Jetha N. Rapport d'étape Recenser les interventions axées sur l'équité visant à promouvoir un poids santé. Promotion de la santé et prévention des maladies chroniques au Canada. 2017;37(3):103-110.

27. Rao DP, Kropac E, Do MT, Roberts KC, Jayaraman GC. Rapport d'étape Embonpoint et obésité chez les enfants au Canada : une évaluation globale. Promotion de la santé et prévention des maladies chroniques au Canada. 2017;37(3):95-102.

28. Steensma C, Loukine L, Choi BCK. Portrait de la compression et de l'expansion de la morbidité au Canada : évolution de l'espérance de vie et de l'espérance de vie ajustée en fonction de la santé, 1994-2010. Promotion de la santé et prévention des maladies chroniques au Canada. 2017;37(3): 74-83.
29. Mayer F, Ross N, Berthelot J, Wilkins R. Espérance de vie sans incapacité selon la région socio-sanitaire. Rapports sur la santé. 2002;13(4):53-66. 